\title{
Factors influencing preparation of polyaniline doped with hydrochloric acid
}

\author{
Sun ChUANYU*, WANG Yu \\ Department of Materials Chemistry, Yinuo Institution, Changchun, 130033, China
}

\begin{abstract}
Factors influencing the reaction of chemical polymerization during aniline doping with hydrochloric acid $(\mathrm{HCl})$ have been studied in this work. The optimal parameters for the preparation of polyaniline were determined as follows: aniline concentration -4 mass \%, molar ratios of oxidant $\left(\mathrm{NH}_{4}\right)_{2} \mathrm{~S}_{2} \mathrm{O}_{8}$ : aniline - 1.2:1 and 1.3:1, the concentration of dopant $-1 \mathrm{~mol} / \mathrm{L}$. Fourier transform infrared spectroscopy (FT-IR) was applied to characterize the structure of polyaniline.
\end{abstract}

Keywords: polyaniline; hydrochloric acid; dopant; conductivity

(C) Wroclaw University of Technology.

\section{Introduction}

Polyaniline (PANI) has been widely investigated since the eighties of the last century due to its easy preparation, environmental stability and low cost [1]. Recently, various potential applications of polyaniline and its derivatives have been studied in such fields as sensors [2], battery anodes [3], electrochemical devices [4]. However, the main drawback of PANI is in itself difficult processing as polyaniline does not dissolve in common solvents and does not diffuse during melting. Researchers found that polyaniline doped with organic acids can be dissolved in common solvents, such as dodecylbenzenesulfonic acid, p-toluene sulfonic acid, camphor sulfonic acid, methanesulfonic acid, and even in water $[5,6]$.

This paper is aiming at the preparation and electrical characterization of polyaniline doped by hydrochloric acid $(\mathrm{HCl})$. Electrical conductivity and productivity of polyaniline were two important factors taken into account on preparing polyaniline with $\mathrm{HCl}$ as dopant [7]. Four aspects, which influence the properties of polyaniline, were studied. The FT-IR spectroscopy was used to analyze the relationship between conductivity and molecular structure of polyaniline products.

*E-mail: chuanyuvip@tom.com

\section{Experiments}

The chemicals used in this experiment were of analytical grade. Aniline was distilled under reduced pressure prior to use. The deionized water was used in all experimental processes.

\subsection{Preparation of polyaniline}

$9.313 \mathrm{~g}(0.100 \mathrm{~mol})$ of aniline was dissolved in $100 \mathrm{~mL}$ of $1 \mathrm{~mol} / \mathrm{L} \mathrm{HCl}$ solution and $28.52 \mathrm{~g}(0.125 \mathrm{~mol})$ of $\left(\mathrm{NH}_{4}\right)_{2} \mathrm{~S}_{2} \mathrm{O}_{8}$ was dissolved in $100 \mathrm{~mL}$ of $1 \mathrm{~mol} / \mathrm{L} \mathrm{HCl}$ solution. Then, $\left(\mathrm{NH}_{4}\right)_{2} \mathrm{~S}_{2} \mathrm{O}_{8}$ solution was slowly droped into the aniline solution for about 45 minutes in an ice bath environment. The solution was kept in the ice bath for 12 hours and the reaction took place in the solution. The resultant product was filtered with distilled water until the filtrate was colorless, then the dried sample was kept under vacuum at $60{ }^{\circ} \mathrm{C}$ for $24 \mathrm{~h}$ to obtain a dark-blue powder.

\subsection{Details of the experiment}

\subsubsection{The effect of concentration of reactant}

In order to ascertain the influence of aniline concentration on electrical conductivity and the yield of polyaniline, different aniline concentrations (mass percent) were used (1\%, $2 \%, 3 \%, 4 \%$, $5 \%, 6 \%, 7 \%, 8 \%, 9 \%, 10 \%)$. The preparation procedure was the same as described in Section 2.1. 
2.2.2. The effect of molar ratio of oxidant:aniline

The molar ratios of $\left(\mathrm{NH}_{4}\right)_{2} \mathrm{~S}_{2} \mathrm{O}_{8}$ :aniline were selected as 0.7:1, 0.9:1, 1.1:1, 1.2:1, 1.3:1, 1.35:1, 1.45:1.

\subsubsection{The effect of dopant concentration}

The concentrations of dopant $(\mathrm{HCl})$ were selected as $0.75 \mathrm{~mol} / \mathrm{L}, 0.7 \mathrm{~mol} / \mathrm{L}, 0.9 \mathrm{~mol} / \mathrm{L}$, $1.5 \mathrm{~mol} / \mathrm{L}, 2 \mathrm{~mol} / \mathrm{L}, 2.5 \mathrm{~mol} / \mathrm{L}, 3.0 \mathrm{~mol} / \mathrm{L}$.

\subsubsection{The effect of ethanol}

In the above reactions, the solvent was made from deionized water and ethanol. The volume ratios of ethanol:deionized water were selected as $0.5: 1,0.75: 1,1: 1,1.5: 1,2.5: 1,3: 1$.

\subsection{Characterization and testing}

\subsubsection{Electrical conductivity}

About $838 \mathrm{mg}$ of the polyaniline product was pressed into a pellet (10 mm diameter, $3 \mathrm{~mm}$ thickness) with a manual hydraulic press at $20 \mathrm{MPa}$. Four-probe method was employed to determine the electrical conductivity of the sample.

\subsubsection{Yield of polyaniline}

The yield of polyaniline was calculated by formula:

$$
y=\frac{m_{\text {polyaniline }}}{m_{\text {aniline }}} \times 100 \%
$$

\subsubsection{FT-IR spectroscopy}

The polyaniline powder was dispersed in potassium bromide $(\mathrm{KBr})$ and then compressed into pellets to obtain FT-IR spectra.

\section{Results and discussion}

\subsection{The influence of concentration of ani- line as reactant}

The concentration of reactant is a key factor influencing a reaction. If a concentration of aniline monomer is higher, the reaction proceeds more violently, which brings about increasing defects in the polymer crystal and producing more oligomers. The electrical conductivity becomes lower and the yield of polyaniline is also at a low level. On the

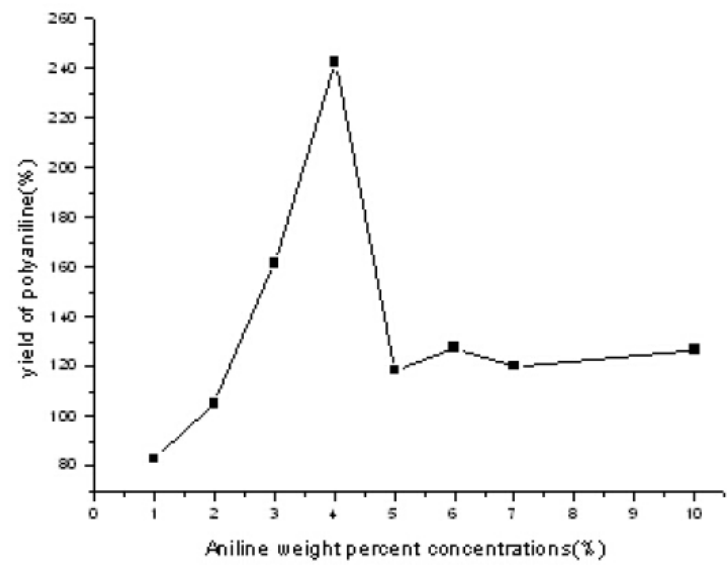

Fig. 1. Effect of monomer concentration on the productivity.

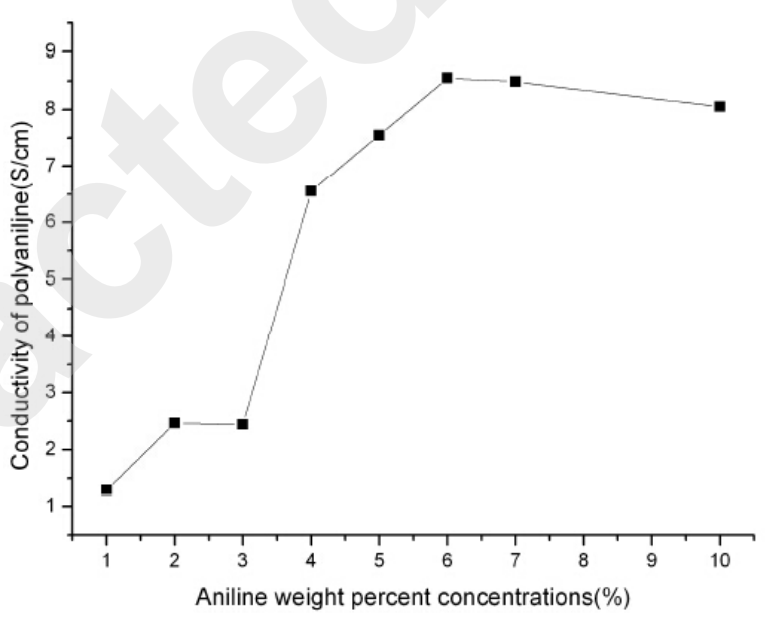

Fig. 2. Effect of monomer concentration on the conductivity.

other hand, if the concentration of monomer aniline is lower, then the oxidation reaction is difficult to initiate. The molecular weight of polyaniline becomes lower, and polymer crystals of poor quality are obtained.

From Fig. 1 and Fig. 2 it can be seen that the electrical conductivity and yield of polyaniline was affected by the concentration of aniline monomer. The highest yield of polyaniline was obtained at mass percentage concentration about $4 \%$. The electrical conductivity of polyaniline almost reached the highest point. 
3.2. The effect of molar ratio of oxidant $\left(\mathbf{N H}_{4}\right)_{2} \mathbf{S}_{2} \mathbf{O}_{8}$ :aniline

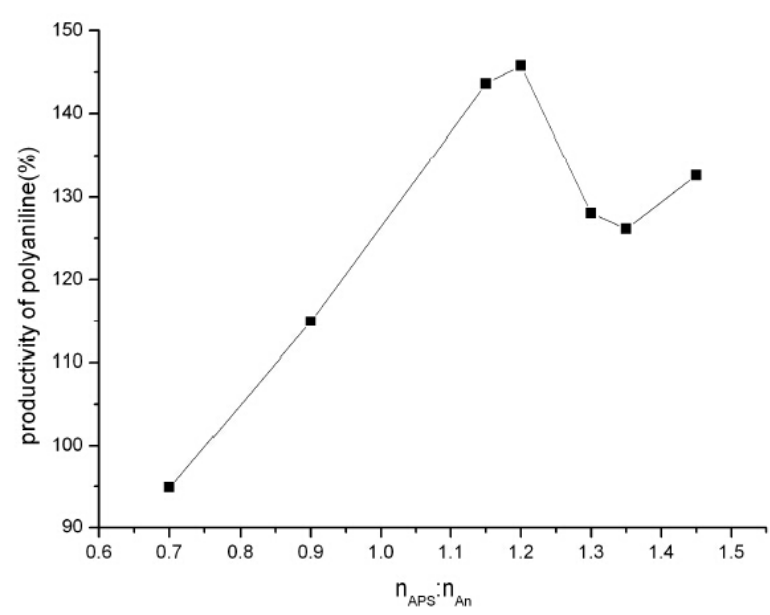

Fig. 3. Effect of oxidant (APS) amount on the productivity.

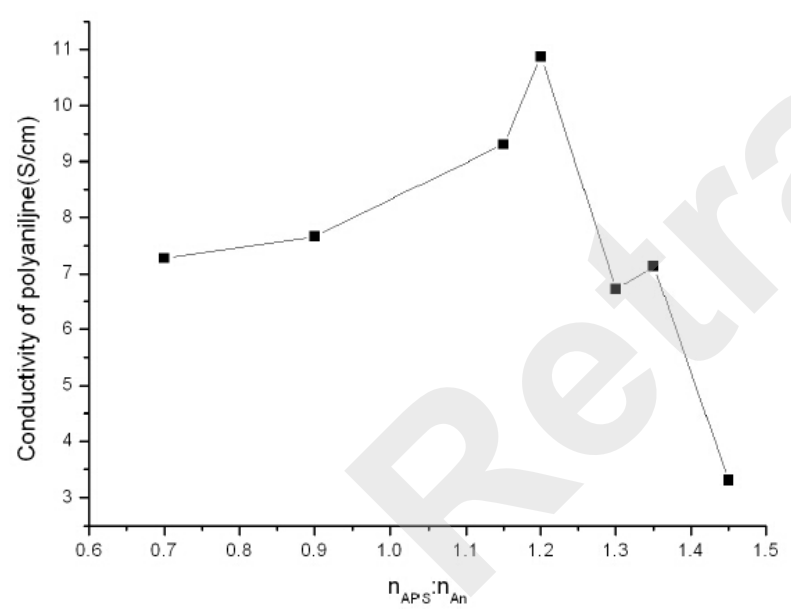

Fig. 4. Effect of oxidant (APS) amount on the conductivity.

Polyaniline molecules have different oxidation states. Only in the middle state polyaniline behaves as a conductor [8]. Therefore, the molar ratio of oxidant $\left(\mathrm{NH}_{4}\right)_{2} \mathrm{~S}_{2} \mathrm{O}_{8}$ and aniline affect the electrical conductivity and yield of polyaniline. Fig. 3 and 4 demonstrate that electrical conductivity and yield of polyaniline reach the optimum point when the mole ratio $n_{\text {oxidant }}: n_{\text {aniline }}$ is $1.25: 1$. For this ratio the yield of polyaniline is $145.8 \%$ and electrical conductivity is $10.87 \mathrm{~S} / \mathrm{cm}$.

\subsection{Effect of the dopant concentration}

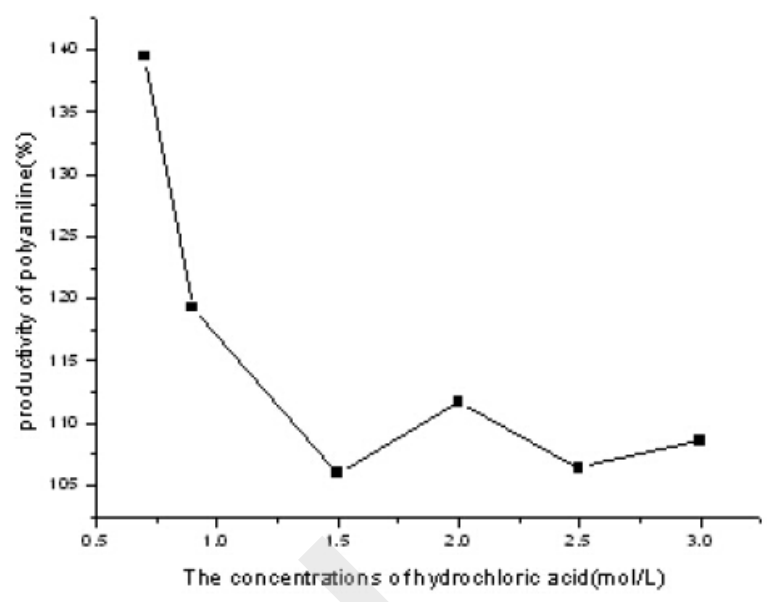

Fig. 5. Influence of $\mathrm{HCl}$ amount on the productivity.

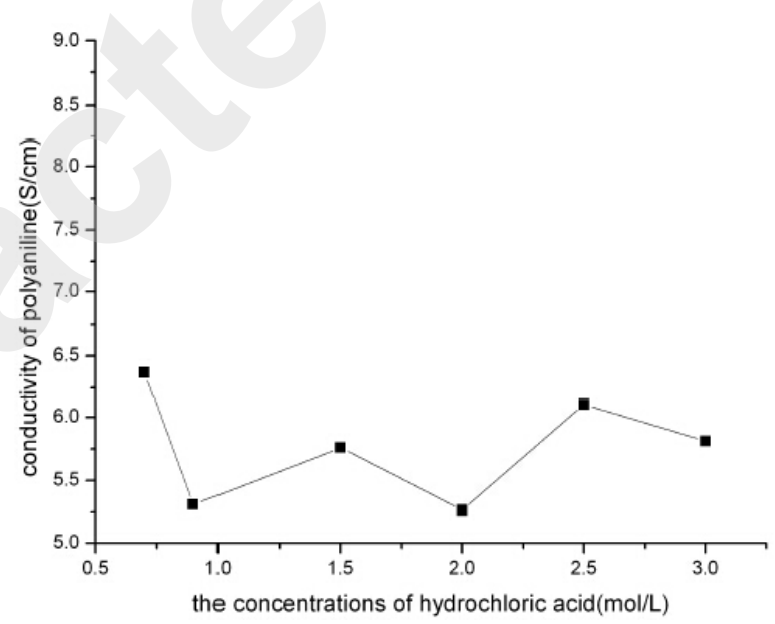

Fig. 6. Influence of $\mathrm{HCl}$ amount on the conductivity.

It can be seen in Fig. 5 that the yield of polyaniline has not changed when the concentration of hydrochloric acid was between $1.5 \mathrm{~mol} / \mathrm{L}$ and $3.0 \mathrm{~mol} / \mathrm{L}$. Sapurina et al. found that polyaniline was possible to obtain only when the $\mathrm{pH}$ value was less than 2, otherwise oligomer was produced [9].

\subsection{The influence of solvent}

In order to control the speed of reaction, organic solvents, such as acetone and chloroform, were added to the reactant, N-Methylpyrrolidone (NMP). All experiments proved that organic solvents had influence on electrical conductivity. 


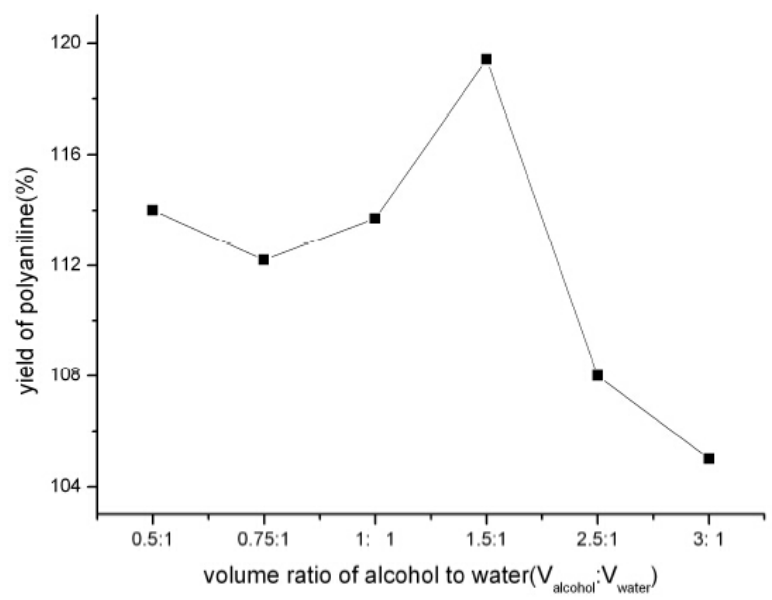

Fig. 7. Influence of volume ratio of alcohol:water on the productivity.

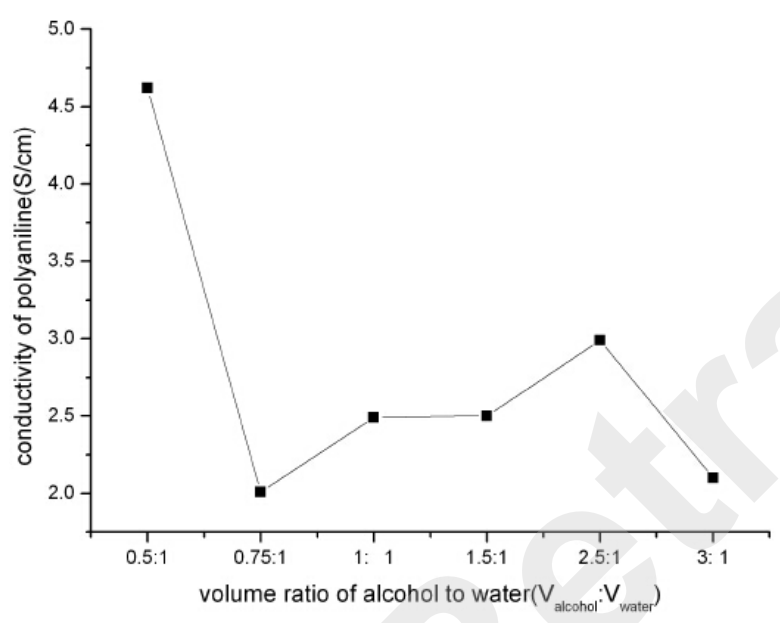

Fig. 8. Influence of volume ratio of alcohol:water on the conductivity.

In the present paper, alcohol was employed as a solvent due to its low price and non-toxicity. As shown in Fig. 7 and 8, the electrical conductivity and yield of polyaniline was affected by alcohol. The yield of polyaniline is the highest at the volume ratio of $\mathrm{V}_{\text {alcohol }}: \mathrm{V}_{\text {water }}=1.5: 1$, and the electrical conductivity of polyaniline reaches the peak when the volume ratio of $\mathrm{V}_{\text {alcohol }}: \mathrm{V}_{\text {water }}$ is $0.5: 1$.

\section{FT-IR investigations}

Fig. 9 presents the FT-IR spectra of polyaniline, which was produced at different molar ratios of

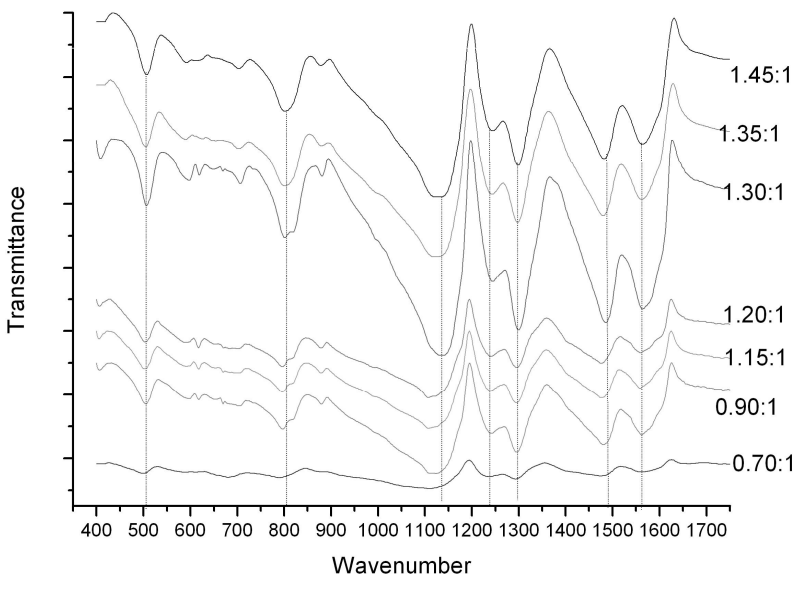

Fig. 9. The FT-IR of PANI produced at different ratios of oxidant and aniline.

oxidant and aniline. The spectra are similar to each other. The band about $1560 \mathrm{~cm}^{-1}$ is assigned to quinonoid rings, while the band near $1481 \mathrm{~cm}^{-1}$ is attributed to the phenyl vibrations. The band of $1298 \mathrm{~cm}^{-1}$ is matched to the N-C-N . The absorption at $1243 \mathrm{~cm}^{-1}$ is assigned $\mathrm{C}-\mathrm{N}$ stretching vibrations. The peaks at $798 \mathrm{~cm}^{-1}$ and $1126 \mathrm{~cm}^{-1}$ are $\mathrm{C}-\mathrm{H}$ bonds $[10,11]$.

According to Fig. 4 the optimal results were achieved at the mole ratio of oxidant to aniline of about 1.20:1 to $1.30: 1$. Previous researchers have found that the electrical conductivity of polyaniline was the highest when polyaniline was at half oxidation state. The transmittance values at the bands near $1560 \mathrm{~cm}^{-1}$ and $1481 \mathrm{~cm}^{-1}$ were similar when the mole ratio of oxidant and aniline was about 1.30:1, which demonstrated that the polyaniline was at the half oxidation state.

\section{Conclusions}

The electrical conductivity and yield of polyaniline are two the most important factors taken into account in the route of preparing polyaniline. Four aspects have been proven to affect the electrical conductivity and yield of polyaniline. The optimal parameters for polyaniline preparation were determined. They are as follows: concentration of aniline - 4 mass $\%$, molar ratio of oxidant $\left(\mathrm{NH}_{4}\right)_{2} \mathrm{~S}_{2} \mathrm{O}_{8}$ :aniline - 1.2:1 to 1.3:1, concentration of dopant $-1 \mathrm{~mol} / \mathrm{L}$, and alcohol used as a solvent. 


\section{References}

[1] Huang Z., Wang P C, Feng J., Synthetic Met., 85 (1) (1997), 1375.

[2] Narang J., Chauhan N., Jain P., Int. J. Biol. Macromol., 50 (3) (2012), 672.

[3] Cheng F., Tang W., Li C., Chem.-Eur. J., 12 (11) (2006), 3082.

[4] Simon P., Gogotsi Y., Nat. Mater., 7 (11) (2008), 845.

[5] Chuanyu S., Yu W., Optoelectron. Adv. M., 6 (11 12) (2012), 1037.

[6] Chuanyu S., M.D. Farabi, Yu W., Optoelectron. Adv. M., 8, (7 - 8) (2014), 810.
[7] Bhadra S., Singha N.K., Khastgir D., Synthetic Met., 156 (16) (2006), 1148.

[8] Albuquerque J.E., Mattoso L.H.C., Balogh D.T., Synthetic Met., 113 (1) (2000), 19.

[9] Sapurina I., Stejskal J., Polym. Int., 57 (12) (2008), 1295.

[10] Chuanyu S., Yu W., Mater. Sci.-Poland, 3 (32) (2014), 419.

[11] Tang J., Jing X., Wang B., Synthetic Met., 24 (3) (1988), 231.

Received 2014-07-03

Accepted 2014-09-04 